\title{
ON THE CAYLEY GRAPHS OF BOOLEAN FUNCTIONS
}

\author{
Lotfallah Pourfaraj* and Modjtaba Ghorbani
}

(C) by University of Niš, Serbia | Creative Commons Licence: CC BY-NC-ND Abstract. A Boolean function is a function $f: \mathbb{Z}_{n}^{2} \rightarrow\{0,1\}$ and we denote the set of all $n$-variable Boolean functions by $B F_{n}$. For $f \in B F_{n}$ the vector $\left[\mathrm{W}_{f}\left(a_{0}\right), \ldots, \mathrm{W}_{f}\left(a_{2 n-1}\right)\right]$ is called the Walsh spectrum of $f$, where $\mathrm{W}_{f}(a)=\sum_{x \in V}(-1)^{f(x) \oplus a x}$, where $V_{n}$ is the vector space of dimension $n$ over the two-element field $F_{2}$. In this paper, we shall consider the Cayley graph $\Gamma_{f}$ associated with a Boolean function $f$. We shall also find a complete characterization of the bent Boolean functions of order 16 and determine the spectrum of related Cayley graphs. In addition, we shall enumerate all orbits of the action of automorphism group on the set $B F_{n}$.

Keywords: Boolean function; Walsh spectrum; Cayley graph; automorphism group.

\section{Introduction}

Suppose $\mathrm{V}_{n}$ is the vector space of dimension $n$ over the two-element field $F_{2}$, namely the set of all $n$-tuples of elements in the field $F_{2}$ and $\oplus$ denotes the addition operator over both $F_{2}$ and the vector space $V_{n}$, where $V_{n}$ is the vector space of dimension $n$ over the two-element field $F_{2}$. A Sylvester-Hadamard matrix of order $2^{n}$ denoted by $H_{n}$ is defined recursively as

$$
H_{0}=1, H_{1}=\left[\begin{array}{cc}
1 & 1 \\
1 & -1
\end{array}\right], \ldots, H_{n}=H_{1} \otimes H_{n-1}, n=1,2, \ldots
$$

where $\otimes$ denotes to Kronecker product or Tensor product.

For two vectors $a, b \in \mathbb{Z}_{2}^{n}$, where $a=\left(a_{1}, \ldots, a_{n}\right)$ and $b=\left(b_{1}, \ldots, b_{n}\right)$, we define their scalar product as $a \cdot b=a_{1} b_{1} \oplus \ldots \oplus a_{n} b_{n}$. A Boolean function $f$ on $n$-variables is a map from $V_{n}$ to $V_{1}$. Suppose the vectors $v_{0}=(0,0, \ldots, 0)$, $v_{1}=(0,0, \ldots, 1), \ldots, v_{2^{n}-1}=(1,1, \ldots, 1)$ are ordered by lexicographical order. The $(0,1)$ sequence $\left(f\left(v_{0}\right), f\left(v_{1}\right), \ldots, f\left(v_{2^{n}-1}\right)\right)$ is called the truth table of $f$ and $B F_{n}$ denotes the set of all $n$-variable Boolean functions.

Received November 13, 2019; accepted February 04, 2020

2010 Mathematics Subject Classification. Primary 06E30; Secondary 05C30

* Corresponding Author 


\section{Walsh spectrum of Boolean functions}

For the Boolean function $f$, the support of $f$ denoted by $\Omega_{f}$ is $\Omega_{f}=\{x \in$ $\left.\mathbb{Z}_{2}^{n}, f(x)=1\right\}$. The Walsh transform of an $n$-variable Boolean function $f$ is an integer valued function $\mathrm{W}_{f}: V_{n} \rightarrow\left[-2^{n}, 2^{n}\right]$ defined by

$$
\mathrm{W}_{f}(u)=\sum_{x \in F_{2}^{n}} f(x)(-1)^{u \cdot x} .
$$

For the Boolean function $f$, the vector $\left[\mathrm{W}_{f}(0), \ldots, \mathrm{W}_{f}\left(2^{n}-1\right)\right]$ is called the Walsh spectrum of $f$, see $[4,7,15,16]$

Consider the Cayley graph $\Gamma_{f}=\operatorname{Cay}\left(\mathbb{Z}_{2}^{n}, \Omega_{f}\right)$, the vertex set of the Cayley graph $\Gamma_{f}$ is $V_{n}$ and two vertices $u, v \in V_{n}$ are adjacent if and only if $f(u \oplus v)=1$. This means that $E_{f}=\left\{(u, v) \mid u, v \in V_{n}, f(u \oplus v)=1\right\}$. Since for every $a \in V_{n}$, $a \oplus a=0$, one can verify that for $\Omega_{f} \subseteq V_{n}$, we have $x=-x \in \Omega_{f}$. We denote this class of Cayley graphs constructed by a Boolean function as B-Cayley graphs. In Appendix $I$, all Boolean functions of order 16 (where $\left|\Omega_{f}\right|=2$ ) and the spectra of B- Cayley graphs are given. In Appendix II, the characterization of Boolean functions in terms of spectrum of B-Cayley graph $\Gamma_{f}$ associated with $f$ is given. In [5], it is proved that for given Boolean function $f$, the Walsh spectrum of B-Cayley graph $\operatorname{Cay}\left(\mathbb{Z}_{2}^{n}, \Omega_{f}\right)$ is equal with $H_{n} . f$. For example, let $f=[1,0,0,1]$ be a Boolean function. Then the Walsh spectrum of B-Cayley graph $\operatorname{Cay}\left(\mathbb{Z}_{2}^{n}, \Omega_{f}\right)$ is

$$
\left(\begin{array}{cccc}
1 & 1 & 1 & 1 \\
1 & -1 & 1 & -1 \\
1 & 1 & -1 & -1 \\
1 & -1 & -1 & 1
\end{array}\right)\left[\begin{array}{l}
1 \\
0 \\
0 \\
1
\end{array}\right]=\left[0^{2}, 2^{2}\right]
$$

For $g=[1,1,1,1]$ we have $H_{n} . g=\left[0^{3}, 4\right]$ and for $h=[0,0,1,1]$ we have $H_{n} . h=$ $\left[-2,0^{2}, 2\right]$.

Theorem 2.1. Let $f$ be a Boolean function whose related B-Cayley graph $\Gamma_{f}$ is a bipartite regular graph with exactly three distinct eigenvalues and -2 is the smallest eigenvalue. Then $f \in F_{2}$ and $f=(0,1,1,0)$.

Proof. Suppose $f$ satisfies in above conditions. We can suppose the spectrum of $\Gamma_{f}$ is $\operatorname{Spec}\left(\Gamma_{f}\right)=\left\{[-2]^{m_{1}},\left[\lambda_{1}\right]^{m_{2}},\left[\lambda_{2}\right]^{m_{3}}\right\}$. Since, $\Gamma_{f}$ is bipartite, $\lambda_{1}=0$ and $\lambda_{1}=2$, see [6]. On the other hand, $\Gamma_{f}$ is regular and so $m_{3}=m_{1}=1$. If $\lambda_{1}, \ldots, \lambda_{n}$ are eigenvalues of a graph, it is a well-known fact that $\sum_{i=1}^{n} \lambda_{i}^{2}=2 \mathrm{~m}$. This implies that $2 m=8$ and so $m=4$. Since $\Gamma_{f}$ is 2-regular, it is isomorphic with the cycle graph $C_{4}$. In addition, suppose $V_{n}=\{00,01,10,11\}$ is the set of vertices of a square as depicted in Figure 2.1. Then we have $00+01=01 \in \Omega_{f}$ and $11+01=10 \in \Omega_{f}$. Hence, $f(00)=f(11)=0$ and $f(01)=f(10)=1$ which yields that $f=(0,1,1,0)$.

Example 1. Suppose $V_{3}=\{000,001,010,100,011,101,110,111\}$. If $a=110$ and $f$ $=(0,0,0,0,1,1,1,10)$, then the related Walsh spectrum is reported in Table 1. 


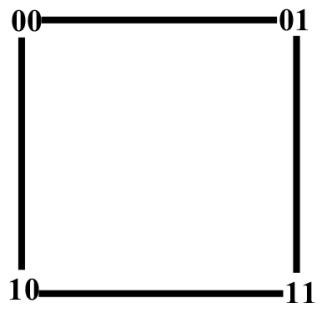

FIG. 2.1: The labeling of vertices of a square.

Table 2.1: The Walsh spectrum of $f=(0,0,0,0,1,1,1,10)$.

\begin{tabular}{c|c|c|c|c|c}
\hline$x$ & $a . x$ & $f$ & $f(x) \oplus a . x$ & $(-1)^{f(x) \oplus a . x}$ & $\mathrm{~W}(f)(a)$ \\
\hline 000 & 0 & 0 & 0 & 1 & \\
001 & 0 & 0 & 0 & 1 & \\
010 & 1 & 0 & 1 & -1 & \\
100 & 1 & 0 & 1 & -1 & 0 \\
011 & 1 & 1 & 0 & 1 & \\
101 & 1 & 1 & 0 & 1 & \\
110 & 0 & 1 & 1 & -1 & \\
111 & 0 & 1 & 1 & -1 & \\
\hline
\end{tabular}

\section{Coloring the B-Cayley graphs}

Let $G$ be a group and $\mathrm{X}$ a nonempty set. An action of $G$ on $\mathrm{X}$ is denoted by $(G \mid \mathrm{X})$ and $\mathrm{X}$ is called a $G$-set. It induces a group homomorphism $\varphi$ from $G$ into the symmetric group $S_{X}$ on $\mathrm{X}$, where $\varphi(g) x=g x$ for all $x \in \mathrm{X}$. The orbit of $x$ will be indicated as $x^{G}$ and defines as the set of all $\varphi(g) x, g \in G$. Suppose $g$ is a permutation of $n$ symbols with exactly $\lambda_{1}$ orbits of size $1, \lambda_{2}$ orbits of size $2, \ldots$, and $\lambda_{n}$ orbits of size $n$. Then the cycle type of $g$ is defined as $1^{\lambda 1} 2^{\lambda 2} \ldots n^{\lambda n}$. Let $G=\mathbb{Z}_{2}^{n}$ and $f \in B F_{n}$ is a Boolean function. In [17] it is showed that the automorphism group $\operatorname{Aut}(G)$ acts on the set $B F_{n}$ as follows:

$$
\forall x_{i} \in \mathbb{Z}_{2}^{n}, \alpha \in \operatorname{Aut}(G): f^{\alpha}\left(x_{i}\right)=f\left(\alpha\left(x_{i}\right)\right) .
$$

Hence, the conjugacy class of $f$ under this action can be computed directly from the definition and it is $[f]=f^{\operatorname{Aut}(G)}=\left\{f^{\alpha}: \alpha \in \operatorname{Aut}(G)\right\}$.

Let $x_{1}, x_{2}, \ldots, x_{n}$ be distinct colors. Denote by $C_{m, n}$ the set of all functions $f$ : $\{1,2, \ldots, m\} \rightarrow\left\{x_{1}, x_{2}, \ldots, x_{n}\right\}$. The action of $p \in S_{m}$ induced on $C_{m, n}$ is defined by $\hat{p}(f)=f o p^{-1}, f \in C_{m, n}$. Treating the colors $x_{1}, x_{2}, \ldots, x_{n}$ that comprise the range of $f \in C_{m, n}$ as independent variables the weight of $f$ is $\mathrm{W}(f)=\Pi_{i} f(i)$. Evidently, $\mathrm{W}(f)$ is a monomial of (total) degree $m$. Suppose $G$ is a permutation group of degree $m, \hat{G}=\{\hat{p}: p \in G\}, \hat{p}$ is as defined above. Let $p_{1}, p_{2}, \ldots, p_{t}$ be the distinct orbits of $\hat{G}$. The weight of $p_{i}$ is the common value of $\mathrm{W}(f), f \in p_{i}$. The sum of the weights of the orbits is the pattern inventory 


$$
\mathrm{W}_{\mathrm{G}}\left(x_{1}, x_{2}, \ldots, x_{n}\right)=\sum_{i=1}^{t} w\left(p_{i}\right) .
$$

Theorem 2 [14]. (Pólya's Theorem) If $G$ is a subgroup of $S_{m}$, then the pattern inventory for the orbits of $C_{m, n}$ modula $\hat{G}$ is

$$
\mathrm{W}_{G}\left(x_{1}, x_{2}, \ldots, x_{n}\right)=\frac{1}{|G|} \sum_{p \in G} M_{1}^{C_{1}(p)} M_{2}^{C_{2}(p)} \ldots M_{m}^{C_{m}(p)}
$$

where $M_{k}=x_{1}^{k}+x_{2}^{k}+\ldots+x_{n}^{k}$, the $k^{\text {th }}$ power sum of the $x$ 's, and $\left(C_{1}(p), \ldots, C_{m}(p)\right)$ is the cycle type of the permutation $p$. We now introduce the notion of cycle index. Let $G$ be a permutation group. The cycle index of $G$ acting on $\mathrm{X}$ is the polynomial $\mathrm{Z}(G, X)$ over $Q$ in terms of in determinates $x_{1}, x_{2}, \ldots, x_{t}, t=|\mathrm{X}|$, defined by

$$
\mathrm{Z}(G, \mathrm{X})=\frac{1}{|G|} \sum_{C \in \operatorname{Conj}(G)}|C| \prod_{i=1}^{t} x_{i}{ }^{C_{i}\left(g_{c}\right)}
$$

where $\operatorname{Conj}(G)$ is the set of all conjugacy classes of $G$ with representatives $g_{C} \in C$.

Let us consider the number of ways of assigning one of the colors green or blue to each corner of a square. Since there are two colors and four corners there are basically $2^{4}=16$ possibilities. However, when we take account of the symmetry of the square we see that some of the possibilities are essentially the same. For example, the first coloring, as in Figure 2 is the same as the second one after rotation through $180^{\circ}$.
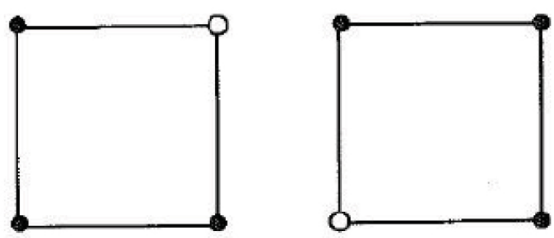

FIG. 3.1: Two indistinguishable colorings.

From above, we regard two colorings as being indistinguishable if one is transformed into the other by symmetry of the square. It is easy to find the distinguishable colorings (in this example) by trial and error: there are just six of them, as shown in the Figure 3.

Now consider a $n$ bead necklace. Let us each corner of it to be colored green or blue. How many different colorings are there? One could argue for $2^{n}$. For example, 


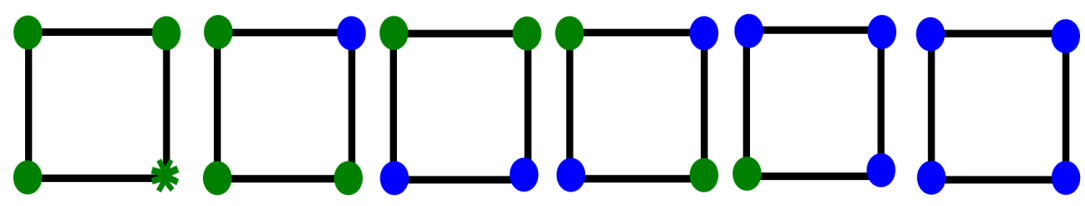

FIG. 3.2: The six distinguishable colorings.

if $n=4$ and the corners are numbered $0,1,2,3$ in clockwise order around the necklace, then there are only 6 ways of coloring the necklace $R R R R, B B B B, R R R B, R B B B$, $R R B B$ and $R B R B$, see Figure 4. On the other hand, all colorings $R B B B, B R B B$, $B B R B, B B B R$ are in the same class. We say that they are equivalent. In other words, the number of all non-equivalent colorings is six. This relation introduces an equivalence relation. All equivalences are

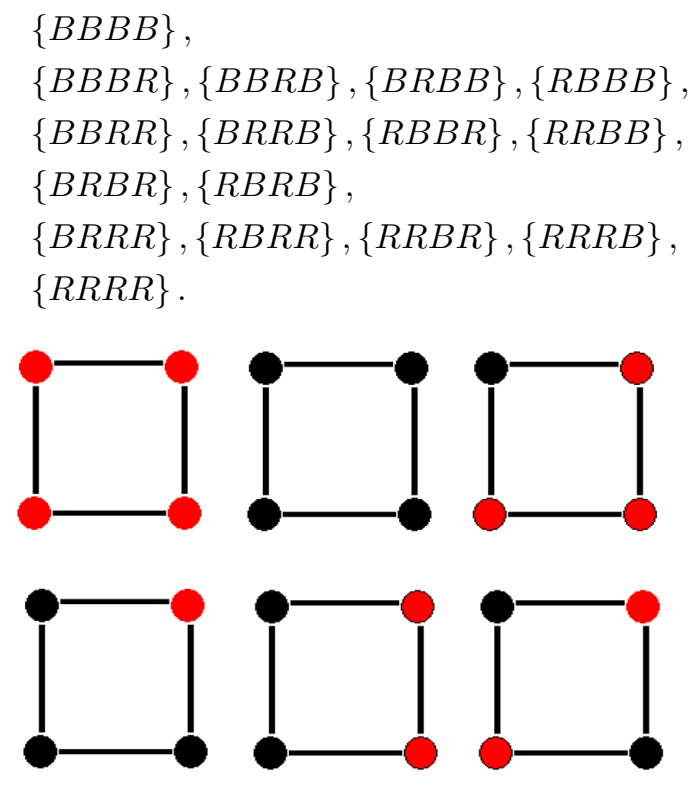

FIG. 3.3: Distinguish colorings of 4 bead necklace.

Hence, any Boolean function can be considered as a coloring of a hyper cube by two colors 0 and 1 . The different colorings yields that there are $2^{2^{n}}$ Boolean functions on $n$ variables.

Definition 3.1. Consider the Boolean function $f$ and B-Cayley $\Gamma_{f}=\operatorname{Cay}\left(\mathbb{Z}_{2}^{n}, \Omega_{f}\right)$. Then $f$ is permutational symmetric (PS) if and only if for any $\left(x_{1}, \ldots, x_{n}\right) \in \mathrm{V}_{n}$, we have $f\left(\alpha\left(x_{1}, \ldots, x_{n}\right)\right)=f\left(x_{1}, \ldots, x_{n}\right)$, for any $\alpha \in \operatorname{Aut}\left(\Gamma_{f}\right)$.

Note that there are $2 n$ different input values corresponding to a function. From the above definition, it is clear that for PS functions, the function $f$ possesses the 
same value corresponding to each of the subsets generated from the automorphism group. As example, for $n=4$, one gets the following partitions:

$$
\begin{aligned}
& \{(0,0,0,0)\}, \\
& \{(0,0,0,1)\},\{(0,0,1,0)\},\{(0,1,0,0)\},\{(1,0,0,0)\}, \\
& \{(0,0,1,1)\},\{(0,1,1,0)\},\{(1,0,0,1)\},\{(1,1,0,0)\}, \\
& \{(0,1,0,1)\},\{(1,0,1,0)\}, \\
& \{(0,1,1,1)\},\{(1,0,1,1)\},\{(1,1,0,1)\},\{(1,1,1,0)\}, \\
& \{(1,1,1,1)\},
\end{aligned}
$$

Therefore, there are six different subsets which partition the 16 input patterns and any 4-variable PS Boolean function can have a specific value corresponding to each subset. If we replace in Eq.(3.3) 0 by $R$ and 1 by $B$, then all above partitions are corresponded to the different colorings of the 2-cube or cycle $\mathrm{C}_{4}$ as given in Eq.(3.2). Hence, there is a 1-1 correspondence between non-equivalent colorings of a $n$-cube and 4 -variable PS Boolean functions. Let us denote

$$
\Lambda_{n}\left(x_{1}, \ldots, x_{n}\right)=\left\{f\left(\alpha\left(x_{1}, \ldots, x_{n}\right)\right)=f\left(x_{1}, \ldots, x_{n}\right): \alpha \in \operatorname{Aut}\left(\Gamma_{f}\right)\right\}
$$

that is, the orbit of $\left(x_{1}, \ldots, x_{n}\right)$ under the action of $\operatorname{Aut}\left(\Gamma_{f}\right)$ on $\mathrm{V}_{n}$. It is clear that $\Lambda_{n}\left(x_{1}, \ldots, x_{n}\right)$ generates a partition in the set $\mathrm{V}_{n}$. Let $\lambda_{n}=\left|\Lambda_{n}\left(x_{1}, \ldots, x_{n}\right)\right|$. It is clear that there are $2^{\lambda_{n}}$ number of $n$-variable PS Boolean functions. Let $\Gamma_{f}$ is B-Cayley graph constructed by given Boolean function $f$. From Polya's Theorem, we get that

$$
\lambda_{n}=\frac{1}{\left|\operatorname{Aut}\left(\Gamma_{f}\right)\right|} \sum_{C \in \operatorname{Conj}(G)}|C| \prod_{i=1}^{t} 2^{C_{i}\left(g_{c}\right)}
$$

in which every variable in Eq. (3.1) is replaced by 2. Hence, we proved the following theorem.

Theorem 3.1. For given Boolean function $f$, the number of PS Boolean functions is

$$
\lambda_{n}=\frac{1}{\left|\operatorname{Aut}\left(\Gamma_{f}\right)\right|} \sum_{C \in \operatorname{Conj}(G)}|C| \prod_{i=1}^{t} 2^{C_{i}\left(g_{c}\right)} .
$$

\section{Application in chemistry: Enumeration of hetero-fullerenes}

Enumeration of chemical compounds has been accomplished by various methods. The Polya-Redfield theorem [14] has been a standard method for combinatorial enumerations of graphs, polyhedra, chemical compounds, and so forth. Combinatorial enumerations have found a wide-ranging application in chemistry, since chemical structural formulas can be regarded as graphs or three-dimensional objects, see [9]. Ghorbani et al. in a series of papers in [1-3,10-12] enumerated the number of hetero-fullerenes with different orders. 
The fullerene era was started in 1985 with the discovery of a stable $\mathrm{C}_{60}$ cluster and its interpretation as a cage structure with the familiar shape of a soccer ball, by Kroto and his co-authors, see $[8,13]$. The well-known fullerene, the $\mathrm{C}_{60}$ molecule, is a closed-cage carbon molecule with three-coordinate carbon atoms tiling the spherical or nearly spherical surface with a truncated icosahedral structure formed by 20 hexagonal and 12 pentagonal rings. Such molecules made up entirely of $n$ carbon atoms and having 12 pentagonal and $(n / 2-10)$ hexagonal faces, where $n \neq 22$ is a natural number equal or greater than 20, see [22-30]. As an application of PolyaTheorem in fullerenes, in Appendix III, the number of hetero-fullerenes of molecule $\mathrm{C}_{60}$ is given.

\section{Acknowledgments}

The results in this paper are part of "Some Properties of Boolean Functions" project. Support of this work was provided for L. Pourfaraj and M. Ghorbani by grant from Islamic Azad University, Central Tehran Branch, Tehran, Iran. In addition, the authors would like to thank Islamic Azad University, Central Tehran Branch, Tehran, Iran. Also the authors would like to thank the referees for their helpful remarks which have contributed to improve the presentation of the article.

\section{RE F E R E N C E S}

1. A. R. Ashrafi and M. Ghorbani: Computer application of GAP to the evaluation of numbers of permutational isomers of hetero fullerenes,. MATCH Commun. Math. Comput. Chem. 60 (2008), 359-367.

2. A. R. Ashrafi, M. Ghorbani and M. Jalali: Detour matrix and detour index of some nanotubes. Digest Journal of Nanomaterials and Biostructures 4 (2008), 245-250.

3. A. R. Ashrafi, M. Jalali, M. Ghorbani and M. V. Diudea: Computing PI and omega polynomials of an infinite family of fullerenes. MATCH Commun. Math. Comput. Chem. 60 (2008), 905-916.

4. M. Bellare, D. Coppersmith, J. Hastad, M. Kiwi and M. Sudan: Linearity testing in characteristic two. IEEE Transactions on Information Theory 42 (1996), 1781-1795.

5. A. Bernasconi and B. Codenotti: Spectral analysis of Boolean functions as a graph eigenvalue problem. IEEE Transactions on Computers 48 (1999), 345-351.

6. N. Biggs: Algebraic Graph Theory. Cambridge Univ. Press, 1968.

7. J. F. Dillon: Elementary Hadamard difference sets. Ph. D. thesis, University of Maryland, 1974.

8. P. W. Fowler and D. E. Manolopoulos, An Atlas of Fullerenes. Oxford Univ. Press, Oxford, 1995.

9. S. Fujita: Symmetry and Combinatorial Enumeration in Chemistry. Springer Verlag, Berlin, 1991.

10. M. Ghorbani: Enumeration of hetero-fullerenes: A survey. MATCH Commun. Math. Comput. Chem. 68 (2012), 381-414. 
11. M. Ghorbani and A. R. Ashrafi: Counting the number of hetero fullerenes. J. Comput. Theor. Nanosci. 3 (2006), 803-810.

12. M. Ghorbani and M. Jalali: Counting numbers of permutational isomers of heterofullerenes. Digest Journal of Nanomaterials and Biostructures, 4 (2008), 269-275.

13. H. W. Kroto, J. R. Heath, S. C. O’Brien, R. F. Curl and R. E. Smalley: buckminsterfullerene. Nature 318 (1985), 162-163.

14. G. PÓlYA and R. C. READ: Combinatorial enumeration of groups and chemical compounds. Springer, New York, 1987.

15. O. S. Rothaus: On bent functions. Journal of Combinatorial Theory 20A (1976), 300-305.

16. P. SARKAR and S. MAITRA: Nonlinearity bounds and constructions of resilient Boolean functions. In Advances in Cryptology-Crypto 2000, LNCS 1807 (2000), 515532.

17. V. Taghvaei-Yazdelli and M. Ghorbani: Characterization of bipartite Cayley graphs in terms of Boolean functions. Journal of Information and Optimization Sciences 39 (2018), 1-18.

Lotfallah Pourfaraj

Faculty of Science

Department of Mathematics

Islamic Azad University

Central Tehran Branch (IAUCTB)

Tehran, I.R. Iran

1.pourfaraj@iauctb.ac.ir

Modjtaba Ghorbani

Department of Mathematics

Faculty of Science

Shahid Rajaee Teacher Training University

Tehran, 16785 - 136, I R. Irana

mghorbani@sru.ac.ir 


\section{Appendix I}

Boolean functions of order 16 where $\Omega_{f}=2$ and spectra of their $B$-Cayley graphs. $f:=[1,1,0,0,0,0,0,0,0,0,0,0,0,0,0,0] \operatorname{Spec}(\mathrm{G}):=[2,0,2,0,2,0,2,0,2,0,2,0,2,0,2,0]$ $f:=[1,0,1,0,0,0,0,0,0,0,0,0,0,0,0,0] \operatorname{Spec}(\mathrm{G}):=[2,2,0,0,2,2,0,0,2,2,0,0,2,2,0,0]$ $f:=[1,0,0,1,0,0,0,0,0,0,0,0,0,0,0,0] \operatorname{Spec}(\mathrm{G}):=[2,0,0,2,2,0,0,2,2,0,0,2,2,0,0,2]$ $f:=[1,0,0,0,1,0,0,0,0,0,0,0,0,0,0,0] \operatorname{Spec}(\mathrm{G}):=[2,2,2,2,0,0,0,0,2,2,2,2,0,0,0,0]$ $f:=[1,0,0,0,0,1,0,0,0,0,0,0,0,0,0,0] \operatorname{Spec}(\mathrm{G}):=[2,0,2,0,0,2,0,2,2,0,2,0,0,2,0,2]$ $f:=[1,0,0,0,0,0,1,0,0,0,0,0,0,0,0,0] \operatorname{Spec}(\mathrm{G}):=[2,2,0,0,0,0,2,2,2,2,0,0,0,0,2,2]$ $f:=[1,0,0,0,0,0,0,1,0,0,0,0,0,0,0,0] \operatorname{Spec}(\mathrm{G}):=[2,0,0,2,0,2,2,0,2,0,0,2,0,2,2,0]$ $f:=[1,0,0,0,0,0,0,0,1,0,0,0,0,0,0,0] \operatorname{Spec}(G):=[2,2,2,2,2,2,2,2,0,0,0,0,0,0,0,0]$ $f:=[1,0,0,0,0,0,0,0,0,1,0,0,0,0,0,0] \operatorname{Spec}(\mathrm{G}):=[2,0,2,0,2,0,2,0,0,2,0,2,0,2,0,2]$ $f:=[1,0,0,0,0,0,0,0,0,0,1,0,0,0,0,0] \operatorname{Spec}(\mathrm{G}):=[2,2,0,0,2,2,0,0,0,0,2,2,0,0,2,2]$ $f:=[1,0,0,0,0,0,0,0,0,0,0,1,0,0,0,0] \operatorname{Spec}(\mathrm{G}):=[2,0,0,2,2,0,0,2,0,2,2,0,0,2,2,0]$ $f:=[1,0,0,0,0,0,0,0,0,0,0,0,1,0,0,0] \mathrm{Spec}(\mathrm{G}):=[2,2,2,2,0,0,0,0,0,0,0,0,2,2,2,2]$ $f:=[1,0,0,0,0,0,0,0,0,0,0,0,0,1,0,0] \operatorname{Spec}(G):=[2,0,2,0,0,2,0,2,0,2,0,2,2,0,2,0]$ $f:=[1,0,0,0,0,0,0,0,0,0,0,0,0,0,1,0] \operatorname{Spec}(\mathrm{G}):=[2,2,0,0,0,0,2,2,0,0,2,2,2,2,0,0]$ $f:=[1,0,0,0,0,0,0,0,0,0,0,0,0,0,0,1] \operatorname{Spec}(G):=[2,0,0,2,0,2,2,0,0,2,2,0,2,0,0,2]$ $f:=[0,1,1,0,0,0,0,0,0,0,0,0,0,0,0,0] \operatorname{Spec}(\mathrm{G}):=[2,0,0,-2,2,0,0,-2,2,0,0,-2,2,0,0,-2]$ $f:=[0,1,0,1,0,0,0,0,0,0,0,0,0,0,0,0] \operatorname{Spec}(\mathrm{G}):=[2,-2,0,0,2,-2,0,0,2,-2,0,0,2,-2,0,0]$ $f:=[0,1,0,0,1,0,0,0,0,0,0,0,0,0,0,0] \operatorname{Spec}(\mathrm{G}):=[2,0,2,0,0,-2,0,-2,2,0,2,0,0,-2,0,-2]$ $f:=[0,1,0,0,0,1,0,0,0,0,0,0,0,0,0,0] \operatorname{Spec}(\mathrm{G}):=[2,-2,2,-2,0,0,0,0,2,-2,2,-2,0,0,0,0]$ $f:=[0,1,0,0,0,0,1,0,0,0,0,0,0,0,0,0] \operatorname{Spec}(\mathrm{G}):=[2,0,0,-2,0,-2,2,0,2,0,0,-2,0,-2,2,0]$ $f:=[0,1,0,0,0,0,0,1,0,0,0,0,0,0,0,0] \operatorname{Spec}(G):=[2,-2,0,0,0,0,2,-2,2,-2,0,0,0,0,2,-2]$ $f:=[0,1,0,0,0,0,0,0,1,0,0,0,0,0,0,0] \operatorname{Spec}(\mathrm{G}):=[2,0,2,0,2,0,2,0,0,-2,0,-2,0,-2,0,-2]$ $f:=[0,1,0,0,0,0,0,0,0,1,0,0,0,0,0,0] \operatorname{Spec}(\mathrm{G}):=[2,-2,2,-2,2,-2,2,-2,0,0,0,0,0,0,0,0]$ $f:=[0,1,0,0,0,0,0,0,0,0,1,0,0,0,0,0] \operatorname{Spec}(\mathrm{G}):=[2,0,0,-2,2,0,0,-2,0,-2,2,0,0,-2,2,0]$ $f:=[0,1,0,0,0,0,0,0,0,0,0,1,0,0,0,0] \operatorname{Spec}(\mathrm{G}):=[2,-2,0,0,2,-2,0,0,0,0,2,-2,0,0,2,-2]$ $f:=[0,1,0,0,0,0,0,0,0,0,0,0,1,0,0,0] \operatorname{Spec}(G):=[2,0,2,0,0,-2,0,-2,0,-2,0,-2,2,0,2,0]$ $f:=[0,1,0,0,0,0,0,0,0,0,0,0,0,1,0,0] \operatorname{Spec}(\mathrm{G}):=[2,-2,2,-2,0,0,0,0,0,0,0,0,2,-2,2,-2]$ $f:=[0,1,0,0,0,0,0,0,0,0,0,0,0,0,1,0] \operatorname{Spec}(G):=[2,0,0,-2,0,-2,2,0,0,-2,2,0,2,0,0,-2]$ $f:=[0,1,0,0,0,0,0,0,0,0,0,0,0,0,0,1] \operatorname{Spec}(G):=[2,-2,0,0,0,0,2,-2,0,0,2,-2,2,-2,0,0]$ $f:=[0,0,1,1,0,0,0,0,0,0,0,0,0,0,0,0] \operatorname{Spec}(\mathrm{G}):=[2,0,-2,0,2,0,-2,0,2,0,-2,0,2,0,-2,0]$ $f:=[0,0,1,0,1,0,0,0,0,0,0,0,0,0,0,0] \operatorname{Spec}(\mathrm{G}):=[2,2,0,0,0,0,-2,-2,2,2,0,0,0,0,-2,-2]$ $f:=[0,0,1,0,0,1,0,0,0,0,0,0,0,0,0,0] \operatorname{Spec}(G):=[2,0,0,-2,0,2,-2,0,2,0,0,-2,0,2,-2,0]$ $f:=[0,0,1,0,0,0,1,0,0,0,0,0,0,0,0,0] \operatorname{Spec}(\mathrm{G}):=[2,2,-2,-2,0,0,0,0,2,2,-2,-2,0,0,0,0]$ $f:=[0,0,1,0,0,0,0,1,0,0,0,0,0,0,0,0] \operatorname{Spec}(G):=[2,0,-2,0,0,2,0,-2,2,0,-2,0,0,2,0,-2]$ $f:=[0,0,1,0,0,0,0,0,1,0,0,0,0,0,0,0] \operatorname{Spec}(\mathrm{G}):=[2,2,0,0,2,2,0,0,0,0,-2,-2,0,0,-2,-2]$ $f:=[0,0,1,0,0,0,0,0,0,1,0,0,0,0,0,0] \operatorname{Spec}(\mathrm{G}):=[2,0,0,-2,2,0,0,-2,0,2,-2,0,0,2,-2,0]$ $f:=[0,0,1,0,0,0,0,0,0,0,1,0,0,0,0,0] \operatorname{Spec}(\mathrm{G}):=[2,2,-2,-2,2,2,-2,-2,0,0,0,0,0,0,0,0]$ $f:=[0,0,1,0,0,0,0,0,0,0,0,1,0,0,0,0] \operatorname{Spec}(\mathrm{G}):=[2,0,-2,0,2,0,-2,0,0,2,0,-2,0,2,0,-2]$ $f:=[0,0,1,0,0,0,0,0,0,0,0,0,1,0,0,0] \operatorname{Spec}(\mathrm{G}):=[2,2,0,0,0,0,-2,-2,0,0,-2,-2,2,2,0,0]$ $f:=[0,0,1,0,0,0,0,0,0,0,0,0,0,1,0,0] \operatorname{Spec}(\mathrm{G}):=[2,0,0,-2,0,2,-2,0,0,2,-2,0,2,0,0,-2]$ $f:=[0,0,1,0,0,0,0,0,0,0,0,0,0,0,1,0] \operatorname{Spec}(\mathrm{G}):=[2,2,-2,-2,0,0,0,0,0,0,0,0,2,2,-2,-2]$ 
$f:=[0,0,1,0,0,0,0,0,0,0,0,0,0,0,0,1] \operatorname{Spec}(\mathrm{G}):=[2,0,-2,0,0,2,0,-2,0,2,0,-2,2,0,-2,0]$ $f:=[0,0,0,1,1,0,0,0,0,0,0,0,0,0,0,0] \operatorname{Spec}(\mathrm{G}):=[2,0,0,2,0,-2,-2,0,2,0,0,2,0,-2,-2,0]$ $f:=[0,0,0,1,0,1,0,0,0,0,0,0,0,0,0,0] \operatorname{Spec}(\mathrm{G}):=[2,-2,0,0,0,0,-2,2,2,-2,0,0,0,0,-2,2]$ $f:=[0,0,0,1,0,0,1,0,0,0,0,0,0,0,0,0] \operatorname{Spec}(\mathrm{G}):=[2,0,-2,0,0,-2,0,2,2,0,-2,0,0,-2,0,2]$ $f:=[0,0,0,1,0,0,0,1,0,0,0,0,0,0,0,0] \operatorname{Spec}(\mathrm{G}):=[2,-2,-2,2,0,0,0,0,2,-2,-2,2,0,0,0,0]$ $f:=[0,0,0,1,0,0,0,0,1,0,0,0,0,0,0,0] \operatorname{Spec}(\mathrm{G}):=[2,0,0,2,2,0,0,2,0,-2,-2,0,0,-2,-2,0]$ $f:=[0,0,0,1,0,0,0,0,0,1,0,0,0,0,0,0] \operatorname{Spec}(G):=[2,-2,0,0,2,-2,0,0,0,0,-2,2,0,0,-2,2]$ $f:=[0,0,0,1,0,0,0,0,0,0,1,0,0,0,0,0] \operatorname{Spec}(\mathrm{G}):=[2,0,-2,0,2,0,-2,0,0,-2,0,2,0,-2,0,2]$ $f:=[0,0,0,1,0,0,0,0,0,0,0,1,0,0,0,0] \operatorname{Spec}(\mathrm{G}):=[2,-2,-2,2,2,-2,-2,2,0,0,0,0,0,0,0,0]$ $f:=[0,0,0,1,0,0,0,0,0,0,0,0,1,0,0,0] \operatorname{Spec}(\mathrm{G}):=[2,0,0,2,0,-2,-2,0,0,-2,-2,0,2,0,0,2]$ $f:=[0,0,0,1,0,0,0,0,0,0,0,0,0,1,0,0] \operatorname{Spec}(\mathrm{G}):=[2,-2,0,0,0,0,-2,2,0,0,-2,2,2,-2,0,0]$ $f:=[0,0,0,1,0,0,0,0,0,0,0,0,0,0,1,0] \operatorname{Spec}(\mathrm{G}):=[2,0,-2,0,0,-2,0,2,0,-2,0,2,2,0,-2,0]$ $f:=[0,0,0,1,0,0,0,0,0,0,0,0,0,0,0,1] \operatorname{Spec}(G):=[2,-2,-2,2,0,0,0,0,0,0,0,0,2,-2,-2,2]$ $f:=[0,0,0,0,1,1,0,0,0,0,0,0,0,0,0,0] \operatorname{Spec}(\mathrm{G}):=[2,0,2,0,-2,0,-2,0,2,0,2,0,-2,0,-2,0]$ $f:=[0,0,0,0,1,0,1,0,0,0,0,0,0,0,0,0] \operatorname{Spec}(\mathrm{G}):=[2,2,0,0,-2,-2,0,0,2,2,0,0,-2,-2,0,0]$ $f:=[0,0,0,0,1,0,0,1,0,0,0,0,0,0,0,0] \operatorname{Spec}(\mathrm{G}):=[2,0,0,2,-2,0,0,-2,2,0,0,2,-2,0,0,-2]$ $f:=[0,0,0,0,1,0,0,0,1,0,0,0,0,0,0,0] \operatorname{Spec}(\mathrm{G}):=[2,2,2,2,0,0,0,0,0,0,0,0,-2,-2,-2,-2]$ $f:=[0,0,0,0,1,0,0,0,0,1,0,0,0,0,0,0] \operatorname{Spec}(\mathrm{G}):=[2,0,2,0,0,-2,0,-2,0,2,0,2,-2,0,-2,0]$ $f:=[0,0,0,0,1,0,0,0,0,0,1,0,0,0,0,0] \operatorname{Spec}(\mathrm{G}):=[2,2,0,0,0,0,-2,-2,0,0,2,2,-2,-2,0,0]$ $f:=[0,0,0,0,1,0,0,0,0,0,0,1,0,0,0,0] \operatorname{Spec}(\mathrm{G}):=[2,0,0,2,0,-2,-2,0,0,2,2,0,-2,0,0,-2]$ $f:=[0,0,0,0,1,0,0,0,0,0,0,0,1,0,0,0] \operatorname{Spec}(\mathrm{G}):=[2,2,2,2,-2,-2,-2,-2,0,0,0,0,0,0,0,0]$ $f:=[0,0,0,0,1,0,0,0,0,0,0,0,0,1,0,0] \operatorname{Spec}(\mathrm{G}):=[2,0,2,0,-2,0,-2,0,0,2,0,2,0,-2,0,-2]$ $f:=[0,0,0,0,1,0,0,0,0,0,0,0,0,0,1,0] \operatorname{Spec}(\mathrm{G}):=[2,2,0,0,-2,-2,0,0,0,0,2,2,0,0,-2,-2]$ $f:=[0,0,0,0,1,0,0,0,0,0,0,0,0,0,0,1] \operatorname{Spec}(\mathrm{G}):=[2,0,0,2,-2,0,0,-2,0,2,2,0,0,-2,-2,0]$ $f:=[0,0,0,0,0,1,1,0,0,0,0,0,0,0,0,0] \operatorname{Spec}(\mathrm{G}):=[2,0,0,-2,-2,0,0,2,2,0,0,-2,-2,0,0,2]$ $f:=[0,0,0,0,0,1,0,1,0,0,0,0,0,0,0,0] \operatorname{Spec}(\mathrm{G}):=[2,-2,0,0,-2,2,0,0,2,-2,0,0,-2,2,0,0]$ $f:=[0,0,0,0,0,1,0,0,1,0,0,0,0,0,0,0] \operatorname{Spec}(\mathrm{G}):=[2,0,2,0,0,2,0,2,0,-2,0,-2,-2,0,-2,0]$ $f:=[0,0,0,0,0,1,0,0,0,1,0,0,0,0,0,0] \operatorname{Spec}(\mathrm{G}):=[2,-2,2,-2,0,0,0,0,0,0,0,0,-2,2,-2,2]$ $f:=[0,0,0,0,0,1,0,0,0,0,1,0,0,0,0,0] \operatorname{Spec}(G):=[2,0,0,-2,0,2,-2,0,0,-2,2,0,-2,0,0,2]$ $f:=[0,0,0,0,0,1,0,0,0,0,0,1,0,0,0,0] \operatorname{Spec}(\mathrm{G}):=[2,-2,0,0,0,0,-2,2,0,0,2,-2,-2,2,0,0]$ $f:=[0,0,0,0,0,1,0,0,0,0,0,0,1,0,0,0] \operatorname{Spec}(\mathrm{G}):=[2,0,2,0,-2,0,-2,0,0,-2,0,-2,0,2,0,2]$ $f:=[0,0,0,0,0,1,0,0,0,0,0,0,0,1,0,0] \operatorname{Spec}(\mathrm{G}):=[2,-2,2,-2,-2,2,-2,2,0,0,0,0,0,0,0,0]$ $f:=[0,0,0,0,0,1,0,0,0,0,0,0,0,0,1,0] \operatorname{Spec}(\mathrm{G}):=[2,0,0,-2,-2,0,0,2,0,-2,2,0,0,2,-2,0]$ $f:=[0,0,0,0,0,1,0,0,0,0,0,0,0,0,0,1] \operatorname{Spec}(\mathrm{G}):=[2,-2,0,0,-2,2,0,0,0,0,2,-2,0,0,-2,2]$ $f:=[0,0,0,0,0,0,1,1,0,0,0,0,0,0,0,0] \operatorname{Spec}(\mathrm{G}):=[2,0,-2,0,-2,0,2,0,2,0,-2,0,-2,0,2,0]$ $f:=[0,0,0,0,0,0,1,0,1,0,0,0,0,0,0,0] \operatorname{Spec}(\mathrm{G}):=[2,2,0,0,0,0,2,2,0,0,-2,-2,-2,-2,0,0]$ $f:=[0,0,0,0,0,0,1,0,0,1,0,0,0,0,0,0] \operatorname{Spec}(\mathrm{G}):=[2,0,0,-2,0,-2,2,0,0,2,-2,0,-2,0,0,2]$ $f:=[0,0,0,0,0,0,1,0,0,0,1,0,0,0,0,0] \operatorname{Spec}(\mathrm{G}):=[2,2,-2,-2,0,0,0,0,0,0,0,0,-2,-2,2,2]$ $f:=[0,0,0,0,0,0,1,0,0,0,0,1,0,0,0,0] \operatorname{Spec}(\mathrm{G}):=[2,0,-2,0,0,-2,0,2,0,2,0,-2,-2,0,2,0]$ $f:=[0,0,0,0,0,0,1,0,0,0,0,0,1,0,0,0] \operatorname{Spec}(\mathrm{G}):=[2,2,0,0,-2,-2,0,0,0,0,-2,-2,0,0,2,2]$ $f:=[0,0,0,0,0,0,1,0,0,0,0,0,0,1,0,0] \operatorname{Spec}(\mathrm{G}):=[2,0,0,-2,-2,0,0,2,0,2,-2,0,0,-2,2,0]$ $f:=[0,0,0,0,0,0,1,0,0,0,0,0,0,0,1,0] \operatorname{Spec}(\mathrm{G}):=[2,2,-2,-2,-2,-2,2,2,0,0,0,0,0,0,0,0]$ $f:=[0,0,0,0,0,0,1,0,0,0,0,0,0,0,0,1] \mathrm{Spec}(\mathrm{G}):=[2,0,-2,0,-2,0,2,0,0,2,0,-2,0,-2,0,2]$ $f:=[0,0,0,0,0,0,0,1,1,0,0,0,0,0,0,0] \operatorname{Spec}(\mathrm{G}):=[2,0,0,2,0,2,2,0,0,-2,-2,0,-2,0,0,-2]$ 
$f:=[0,0,0,0,0,0,0,1,0,1,0,0,0,0,0,0] \operatorname{Spec}(G):=[2,-2,0,0,0,0,2,-2,0,0,-2,2,-2,2,0,0]$ $f:=[0,0,0,0,0,0,0,1,0,0,1,0,0,0,0,0] \operatorname{Spec}(\mathrm{G}):=[2,0,-2,0,0,2,0,-2,0,-2,0,2,-2,0,2,0]$ $f:=[0,0,0,0,0,0,0,1,0,0,0,1,0,0,0,0] \operatorname{Spec}(\mathrm{G}):=[2,-2,-2,2,0,0,0,0,0,0,0,0,-2,2,2,-2]$ $f:=[0,0,0,0,0,0,0,1,0,0,0,0,1,0,0,0] \operatorname{Spec}(G):=[2,0,0,2,-2,0,0,-2,0,-2,-2,0,0,2,2,0]$ $f:=[0,0,0,0,0,0,0,1,0,0,0,0,0,1,0,0] \operatorname{Spec}(\mathrm{G}):=[2,-2,0,0,-2,2,0,0,0,0,-2,2,0,0,2,-2]$ $f:=[0,0,0,0,0,0,0,1,0,0,0,0,0,0,1,0] \operatorname{Spec}(\mathrm{G}):=[2,0,-2,0,-2,0,2,0,0,-2,0,2,0,2,0,-2]$ $f:=[0,0,0,0,0,0,0,1,0,0,0,0,0,0,0,1] \operatorname{Spec}(G):=[2,-2,-2,2,-2,2,2,-2,0,0,0,0,0,0,0,0]$ $f:=[0,0,0,0,0,0,0,0,1,1,0,0,0,0,0,0] \operatorname{Spec}(G):=[2,0,2,0,2,0,2,0,-2,0,-2,0,-2,0,-2,0]$ $f:=[0,0,0,0,0,0,0,0,1,0,1,0,0,0,0,0] \operatorname{Spec}(G):=[2,2,0,0,2,2,0,0,-2,-2,0,0,-2,-2,0,0]$ $f:=[0,0,0,0,0,0,0,0,1,0,0,1,0,0,0,0] \operatorname{Spec}(\mathrm{G}):=[2,0,0,2,2,0,0,2,-2,0,0,-2,-2,0,0,-2]$ $f:=[0,0,0,0,0,0,0,0,1,0,0,0,1,0,0,0] \operatorname{Spec}(\mathrm{G}):=[2,2,2,2,0,0,0,0,-2,-2,-2,-2,0,0,0,0]$ $f:=[0,0,0,0,0,0,0,0,1,0,0,0,0,1,0,0] \operatorname{Spec}(\mathrm{G}):=[2,0,2,0,0,2,0,2,-2,0,-2,0,0,-2,0,-2]$ $f:=[0,0,0,0,0,0,0,0,1,0,0,0,0,0,1,0] \operatorname{Spec}(G):=[2,2,0,0,0,0,2,2,-2,-2,0,0,0,0,-2,-2]$ $f:=[0,0,0,0,0,0,0,0,1,0,0,0,0,0,0,1] \operatorname{Spec}(\mathrm{G}):=[2,0,0,2,0,2,2,0,-2,0,0,-2,0,-2,-2,0]$ $f:=[0,0,0,0,0,0,0,0,0,1,1,0,0,0,0,0] \operatorname{Spec}(G):=[2,0,0,-2,2,0,0,-2,-2,0,0,2,-2,0,0,2]$ $f:=[0,0,0,0,0,0,0,0,0,1,0,1,0,0,0,0] \operatorname{Spec}(G):=[2,-2,0,0,2,-2,0,0,-2,2,0,0,-2,2,0,0]$ $f:=[0,0,0,0,0,0,0,0,0,1,0,0,1,0,0,0] \operatorname{Spec}(\mathrm{G}):=[2,0,2,0,0,-2,0,-2,-2,0,-2,0,0,2,0,2]$ $f:=[0,0,0,0,0,0,0,0,0,1,0,0,0,1,0,0] \operatorname{Spec}(\mathrm{G}):=[2,-2,2,-2,0,0,0,0,-2,2,-2,2,0,0,0,0]$ $f:=[0,0,0,0,0,0,0,0,0,1,0,0,0,0,1,0] \operatorname{Spec}(\mathrm{G}):=[2,0,0,-2,0,-2,2,0,-2,0,0,2,0,2,-2,0]$ $f:=[0,0,0,0,0,0,0,0,0,1,0,0,0,0,0,1] \operatorname{Spec}(\mathrm{G}):=[2,-2,0,0,0,0,2,-2,-2,2,0,0,0,0,-2,2]$ $f:=[0,0,0,0,0,0,0,0,0,0,1,1,0,0,0,0] \operatorname{Spec}(\mathrm{G}):=[2,0,-2,0,2,0,-2,0,-2,0,2,0,-2,0,2,0]$ $f:=[0,0,0,0,0,0,0,0,0,0,1,0,1,0,0,0] \operatorname{Spec}(\mathrm{G}):=[2,2,0,0,0,0,-2,-2,-2,-2,0,0,0,0,2,2]$ $f:=[0,0,0,0,0,0,0,0,0,0,1,0,0,1,0,0] \operatorname{Spec}(G):=[2,0,0,-2,0,2,-2,0,-2,0,0,2,0,-2,2,0]$ $f:=[0,0,0,0,0,0,0,0,0,0,1,0,0,0,1,0] \operatorname{Spec}(\mathrm{G}):=[2,2,-2,-2,0,0,0,0,-2,-2,2,2,0,0,0,0]$ $f:=[0,0,0,0,0,0,0,0,0,0,1,0,0,0,0,1] \operatorname{Spec}(\mathrm{G}):=[2,0,-2,0,0,2,0,-2,-2,0,2,0,0,-2,0,2]$ $f:=[0,0,0,0,0,0,0,0,0,0,0,1,1,0,0,0] \operatorname{Spec}(\mathrm{G}):=[2,0,0,2,0,-2,-2,0,-2,0,0,-2,0,2,2,0]$ $f:=[0,0,0,0,0,0,0,0,0,0,0,1,0,1,0,0] \operatorname{Spec}(\mathrm{G}):=[2,-2,0,0,0,0,-2,2,-2,2,0,0,0,0,2,-2]$ $f:=[0,0,0,0,0,0,0,0,0,0,0,1,0,0,1,0] \operatorname{Spec}(\mathrm{G}):=[2,0,-2,0,0,-2,0,2,-2,0,2,0,0,2,0,-2]$ $f:=[0,0,0,0,0,0,0,0,0,0,0,1,0,0,0,1] \operatorname{Spec}(\mathrm{G}):=[2,-2,-2,2,0,0,0,0,-2,2,2,-2,0,0,0,0]$ $f:=[0,0,0,0,0,0,0,0,0,0,0,0,1,1,0,0] \operatorname{Spec}(\mathrm{G}):=[2,0,2,0,-2,0,-2,0,-2,0,-2,0,2,0,2,0]$ $f:=[0,0,0,0,0,0,0,0,0,0,0,0,1,0,1,0] \operatorname{Spec}(\mathrm{G}):=[2,2,0,0,-2,-2,0,0,-2,-2,0,0,2,2,0,0]$ $f:=[0,0,0,0,0,0,0,0,0,0,0,0,1,0,0,1] \operatorname{Spec}(\mathrm{G}):=[2,0,0,2,-2,0,0,-2,-2,0,0,-2,2,0,0,2]$ $f:=[0,0,0,0,0,0,0,0,0,0,0,0,0,1,1,0] \operatorname{Spec}(\mathrm{G}):=[2,0,0,-2,-2,0,0,2,-2,0,0,2,2,0,0,-2]$ $f:=[0,0,0,0,0,0,0,0,0,0,0,0,0,1,0,1] \operatorname{Spec}(G):=[2,-2,0,0,-2,2,0,0,-2,2,0,0,2,-2,0,0]$ $f:=[0,0,0,0,0,0,0,0,0,0,0,0,0,0,1,1] \operatorname{Spec}(\mathrm{G}):=[2,0,-2,0,-2,0,2,0,-2,0,2,0,2,0,-2,0]$ 
Appendix II: All Boolean functions of order 16 .

\begin{tabular}{|c|c|}
\hline Boolean function $f$ & Eigenvalues \\
\hline$(0,1,0,0,0,0,0,0,0,0,1,0,0,0,0,0)$ & $-2^{4}, 0^{8}, 2^{4}$ \\
\hline$(0,1,1,1,0,0,0,0,0,0,0,0,0,0,0,0)$ & $-1^{12}, 3^{4}$ \\
\hline$(0,1,1,0,1,0,0,0,0,0,0,0,0,0,0,0)$ & $-3^{2},-1^{6}, 1^{6}, 3^{2}$ \\
\hline$(0,1,0,0,1,1,0,0,0,0,0,0,0,0,0,0)$ & $-1^{12}, 3^{4}$ \\
\hline$(0,1,1,1,1,0,0,0,0,0,0,0,0,0,0,0)$ & $-2^{6}, 0^{6}, 2^{2}, 4^{2}$ \\
\hline$(0,1,1,0,0,1,1,0,0,0,0,0,0,0,0,0)$ & $-4^{2}, 0^{12}, 4^{2}$ \\
\hline$(0,1,1,0,0,0,1,0,0,0,0,0,0,1,0,0)$ & $-4,-2^{4}, 0^{6}, 2^{4}, 4$ \\
\hline$(0,1,1,1,1,1,0,0,0,0,0,0,0,0,0,0)$ & $-3^{2},-1^{8}, 1^{4}, 5^{2}$ \\
\hline$(0,1,1,1,1,0,0,0,1,0,0,0,0,0,0,0)$ & $-3^{2},-1^{8}, 1^{4}, 5^{2}$ \\
\hline$(0,1,1,1,1,0,0,0,1,0,0,0,0,0,0,0)$ & $-3^{3} \cdot-1^{6}, 1^{4}, 3^{2}, 5$ \\
\hline$(0,1,1,0,1,0,0,1,0,1,0,0,0,0,0,0)$ & $-5,-3,-1^{6}, 1^{6}, 3,5$ \\
\hline$(0,1,0,1,1,0,0,0,1,0,0,0,0,0,1,0)$ & $-3^{5}, 1^{10}, 5$ \\
\hline$(0,1,1,1,1,1,0,0,1,0,0,0,0,0,0,0)$ & $-4,-2^{5}, 0^{6}, 2^{2}, 4,6$ \\
\hline$(0,1,1,1,1,0,0,0,1,0,0,0,1,0,0,0)$ & $-2^{9}, 2^{6}, 6$ \\
\hline$(0,1,1,1,1,0,0,0,1,0,0,0,0,1,0,0)$ & $-4^{2},-2^{3}, 2^{4}, 0^{6}, 6$ \\
\hline$(0,1,0,0,1,0,1,0,1,0,1,0,0,1,0,0)$ & $-6,-2^{3}, 0^{8}, 2^{3}, 6$ \\
\hline$(0,1,0,0,0,0,1,1,0,0,1,1,1,0,0,0)$ & $-2^{6}, 0^{8}, 6^{2}$ \\
\hline$(0,1,1,1,1,1,1,1,0,0,0,0,0,0,0,0)$ & $-1^{14}, 7^{2}$ \\
\hline$(0,1,1,1,1,1,1,0,1,0,0,0,0,0,0,0)$ & $-3^{2},-1^{7}, 1^{4}, 5,7$ \\
\hline$(0,1,1,1,1,1,0,0,1,1,0,0,0,0,0,0)$ & $-5,-1^{11}, 3^{3}, 7$ \\
\hline$(0,1,1,1,1,1,0,0,1,0,1,0,0,0,0,0)$ & $-3^{4},-1^{5}, 1^{4}, 3^{2}, 7$ \\
\hline$(0,1,1,1,1,1,0,0,1,0,0,0,0,0,0,1)$ & $-5,-3^{2},-1^{5}, 1^{6}, 3,7$ \\
\hline$(0,0,0,0,0,0,0,0,1,0,1,1,1,1,1,1)$ & $-7,-1^{7}, 1^{7}, 7$ \\
\hline$(0,1,1,1,1,1,1,1,0,1,0,0,0,0,0,0)$ & $-2^{7}, 0^{7}, 6,8$ \\
\hline$(0,1,1,1,1,1,1,0,1,1,0,0,0,0,0,0)$ & $-4,-2^{6}, 0^{5}, 2^{2}, 4,8$ \\
\hline$(0,1,1,1,1,1,1,0,1,0,0,0,0,0,0,1)$ & $-4^{3}, 0^{11}, 4,8$ \\
\hline$(0,1,1,1,1,1,0,0,1,1,0,0,0,0,1,0)$ & $-6,-2^{4}, 0^{7}, 2^{3}, 8$ \\
\hline$(0,1,0,1,1,0,1,0,1,0,1,0,0,1,0,1)$ & $-8,0^{14}, 8$ \\
\hline$(0,1,1,1,1,1,1,1,1,1,0,0,0,0,0,0)$ & $-3^{3},-1^{8}, 1^{3}, 5,9$ \\
\hline$(0,1,1,1,1,1,1,0,1,0,1,1,0,0,0,0)$ & $-3^{4},-1^{6}, 1^{3}, 3^{2}, 9$ \\
\hline$(0,1,1,1,1,1,1,0,1,1,0,0,0,0,1,0)$ & $-5,-3^{2},-1^{6}, 1^{5}, 3,9$ \\
\hline$(0,1,1,0,1,0,1,1,1,0,1,1,0,0,0,1)$ & $-3^{6}, 1^{9}, 9$ \\
\hline$(0,1,1,0,0,1,1,1,1,0,0,1,1,0,0,1)$ & $-7,-1^{8}, 1^{6}, 9$ \\
\hline$(0,1,0,1,1,1,1,0,1,1,0,1,0,0,0,1)$ & $-3^{6}, 1^{9}, 9$ \\
\hline$(0,1,1,1,1,1,1,1,1,1,0,1,0,0,0,0)$ & $-4,-2^{6}, 0^{6}, 2,4,10$ \\
\hline$(0,1,1,1,1,1,1,0,1,1,0,1,0,1,0,0)$ & $-2^{10}, 2^{5}, 10$ \\
\hline$(0,1,1,1,1,1,1,0,1,1,0,0,0,0,1,1)$ & $-6,-2^{4}, 0^{8}, 2^{2}, 10$ \\
\hline$(0,1,1,1,1,1,1,0,1,0,1,0,1,0,0,1)$ & $-4^{2},-2^{4}, 0^{6}, 2^{3}, 10$ \\
\hline$(0,1,1,1,1,1,1,1,1,1,1,1,0,0,0,0)$ & $-5,-1^{12}, 3^{2}, 11$ \\
\hline$(0,1,1,1,1,1,1,1,1,1,1,0,1,0,0,0)$ & $-3^{4},-1^{6}, 1^{4}, 3,11$ \\
\hline$(0,1,1,1,1,1,1,0,1,1,1,0,0,0,1,1)$ & $-5,-3^{2},-1^{6}, 1^{6}, 11$ \\
\hline$(0,1,1,1,1,1,1,1,1,1,1,1,1,0,0,0)$ & $-4,-2^{6}, 0^{6}, 2,12$ \\
\hline$(0,1,1,1,1,1,1,0,1,1,1,0,0,1,1,1)$ & $-4^{3}, 0^{12}, 12$ \\
\hline$(0,1,1,1,1,1,1,1,1,1,1,1,1,1,0,0)$ & $-3^{3},-1^{8}, 1^{4}, 13$ \\
\hline$(0,1,1,1,1,1,1,1,0,1,1,1,1,1,1,1)$ & $-2^{7}, 0^{8}, 14$ \\
\hline$(0,1,1,1,1,1,1,1,1,1,1,1,1,1,1,1)$ & $-1^{15}, 15$ \\
\hline
\end{tabular}


Appendix III: The number of $C_{60-k} B_{k}$ molecules.

\begin{tabular}{l|l}
\hline$k, 60-k$ & Number of hetero-fullerenes $C_{60-k} B_{k}$ \\
\hline 0,60 & 1 \\
1,59 & 1 \\
2,58 & 37 \\
3,57 & 577 \\
4,56 & 8236 \\
5,55 & 91030 \\
6,54 & 835476 \\
7,53 & 6436782 \\
8,52 & 42650532 \\
9,51 & 246386091 \\
10,50 & 1256602779 \\
11,49 & 5711668755 \\
12,48 & 23322797475 \\
13,47 & 86114390460 \\
14,46 & 289098819780 \\
15,45 & 886568158468 \\
16,44 & 2493474394140 \\
17,43 & 6453694644705 \\
18,42 & 15417163018725 \\
19,41 & 34080036632565 \\
20,40 & 69864082608210 \\
21,39 & 133074428781570 \\
22,38 & 235904682814710 \\
23,37 & 389755540347810 \\
24,36 & 600873146368170 \\
25,35 & 865257299572455 \\
26,34 & 1164769471671687 \\
27,33 & 1466746704458899 \\
28,32 & 1728665795116244 \\
29,31 & 1907493251046152 \\
30,30 & 1971076398255692 \\
\hline
\end{tabular}

